\title{
Complete Genome Resource of Pseudomonas coronafaciens X-1 Causing Halo Blight Disease in Oats
}

Young Cheol Kim ${ }^{\dagger}$

Department of Applied Biology, Chonnam National University, Gwangju 61186, Republic of Korea

\begin{abstract}
Pseudomonas coronafaciens X-1 was isolated from halo blight lesions on oat leaves in Korea. Currently, no genome resource on the oat halo blight pathogen is available. Here, the complete genome sequence of this strain, containing a circular chromosome and one circular megaplasmid is reported. This is the first genome sequence of the oat halo blight pathogen and will contribute to our understanding of the pathogenicity and ecological aspects of the halo blight pathogen and the interactions between $P$. coronafaciens and oats.
\end{abstract}

\section{Genome Announcement}

Strains classified in Pseudomonas coronafaciens are important pathogens of oats (Elliot 1920; Griffiths and Peregrine, 1956; Wilkie 1972). This is a gram-negative bacterium pathogenic to plants and is classified as Pseudomonas syringae group genomosp. 4 (Hwang et al. 2005). A recent study indicated that $P$. coronafaciens strains also cause yellow bud diseases in onion (Dutta et al. 2018). Consequently, Pseudomonas coronafaciens sp. nov. is proposed as a new species in genus Pseudomonas that is distinct from $P$. syringae (Dutta et al. 2018). Among the $P$. coronafaciens strains, the complete genome sequence of only $P$. coronafaciens pv. oryzae strain 1_6, a rice pathogen, has recently been completed (Baltrus and Clark, 2020). However, at present, no genome sequence of the oat halo blight pathogen is available. In this study, we report the complete genome sequence of $P$. coronafaciens $\mathrm{X}-1$, which causes halo blight in oats.

Recently, we isolated a strain of the oat halo blight pathogen from oats grown in South Korea (Kim 2020). The pure culture of the $P$. coronafaciens $X-1$ strain was grown overnight in King's B medium and was stored at $-80^{\circ} \mathrm{C}$ in $25 \%$ glycerol as a stock culture. The strain was obtained by single-colony isolation on King's $B$ agar plates and was aerobically cultured in King's B broth at $20^{\circ} \mathrm{C}$ for 2 days. Genomic DNA was extracted using a genomic DNA kit (Macrogen), following manufacturer protocols, with RNase A treatment. The quality and quantity of the DNA was evaluated via a fluorescence-based quantification method using the Quant-iT PicoGreen dsDNA assay kit (Thermo Fisher Scientific Korea Ltd.) and Victor 3 fluorometry (PerkinElmer). Genomic DNA was sequenced by Macrogen Inc. (Seoul, South Korea) on single-molecule real-time (SMRT) cells using PacBio RS II SMRT sequencing technology (Pacific Biosciences) as well as using a HiSeq X-ten platform (Illumina). The sequencing libraries were prepared using a DNA template prep kit v3.0 for PacBio RS II and a TruSeq Nano DNA kit for Illumina, according to manufacturer protocols. All software systems were run using their default settings, unless otherwise noted. After subread filtering of the raw

\footnotetext{
${ }^{\dagger}$ Corresponding author: Y. C. Kim: yckimyc@jnu.ac.kr
}

The author(s) declare no conflict of interest.

Accepted for publication 6 June 2020.

(C) 2020 The American Phytopathological Society

\section{Funding}

This work was supported by the Cooperative Research Program for Agriculture Science \& Technology Development, Rural Development Administration, Republic of Korea (Project No. PJ01499503).

\section{Keywords}

bacterial halo blight, genomics, Pseudomonas coronafaciens 
Table 1. Genomic features of Pseudomonas coronafaciens

\begin{tabular}{|c|c|c|c|c|}
\hline \multirow[b]{2}{*}{ Genomic features } & \multicolumn{2}{|c|}{ P. coronafaciens $\mathrm{X}-1$} & \multicolumn{2}{|c|}{ P. coronafaciens pv. oryzae 1_6 } \\
\hline & Contig 1 (chromosome) & Contig 2 (megaplasmid) & Contig 1 (chromosome) & Contig 2 (megaplasmid) \\
\hline Molecular shape & Circular & Circular & Circular & Circular \\
\hline GenBank accession number & CP050260 & СР050261 & СР046035 & СР046036 \\
\hline Genome length (bp) & $5,661,408$ & 94,384 & $5,662,937$ & 51,521 \\
\hline Total coding sequence & 5,150 & 118 & 4,964 & 54 \\
\hline $\mathrm{G}+\mathrm{C}$ content $(\%)$ & 57.80 & 52.87 & 57.80 & 54.64 \\
\hline Ribosomal RNAs & 16 & & 8 & \\
\hline Transfer RNAs & 66 & & 63 & \\
\hline Reference & \multicolumn{2}{|c|}{ This study } & \multicolumn{2}{|c|}{ Baltrus and Clark 2020} \\
\hline
\end{tabular}

data, a total of 142,108 subreads $\left(N_{50}\right.$ value was 14,444$)$ with an average length of $10,189 \mathrm{bp}$ (total 1,447,960,463 bp) provided approximately 300-fold genome coverage. The subreads were de novo assembled using the Canu v1.3 assembler (Koren et al. 2017), and the overlapping regions at both ends of each contig were trimmed to generate unique stretches on both ends using Circlator (Hunt et al. 2015). These subreads were assembled using the RS hierarchical genome assembly process (HGAP v3.0) and SMRT Portal (v2.3) de novo assembler (Chin et al. 2013.). After the de novo assembly step, error correction was conducted by making complete genome contigs with Illumina reads for sequencing compensation for any inaccuracies introduced by PacBio sequencing. The error-corrected assemblies were further polished using Pilon v1.22 (Walker et al. 2014) with trimmed paired-end reads. After the complete genome was assembled, BLAST analysis was performed to identify the species that showed similarity to each scaffold. To assess the completeness of the genome assembly, the benchmarking universal single-copy orthologous (BUSCO v3.0) (Seppey et al. 2019) analysis was performed, based on evolutionarily informed expectations of gene content from near-universal single-copy orthologs. By default, bacterial_odb9 DB was used for analysis. The BUSCO analysis showed that 146 complete and single-copy BUSCOs (98.65\%) and two missing BUSCOs (1.35\%) were detected. The genome was annotated using Prokka v1.12b (Seemann, 2014) as a pipeline that performed a series of processes automatically.

The genome of $P$. coronafaciens $\mathrm{X}-1$ comprised two large circular replicons with one chromosome and one megaplasmid. The genome features of the $X-1$ strain are summarized in Table 1. The chromosome of X-1 was 5,661,408 bp in length with 5,150 protein-encoding genes, a $57.8 \% \mathrm{G}+\mathrm{C}$ content, 16 ribosomal RNAs, and 66 transfer RNAs. The megaplasmid was 94,385 bp long with 118 protein-encoding genes and a $52.87 \% \mathrm{G}+\mathrm{C}$ content (Table 1). This complete genome sequence of $P$. coronafaciens $\mathrm{X}-1$ will provide a valuable resource for future studies on the oat halo blight pathogen and its interactions with oats.

\section{Data Availability}

A pure culture of $P$. coronafaciens X-1, which was sequenced in this study, is available at the Korean Agricultural Culture Collection (KACC19998181), National Agrobiodiversity Center, Wanju, South Korea. This genome project is indexed at GenBank under BioProject accession number PRJNA613155 and Bio Sample ID SAMN14389309. The chromosomal sequence for $P$. coronafaciens $X-1$ can be found at GenBank under accession number CP050260, whereas the sequence for the plasmid can be found under accession number CP050261.

\section{Literature Cited}

Baltrus, D. A., and Clark, M. 2020. Complete genome sequence of Pseudomonas coronafaciens pv. oryzae 1_6. Microbiol. Resour. Announc. 9:e01564-19.

Chin, C.-S., Alexander, D. H., Marks, P., Klammer, A. A., Drake, J., Heiner, C., Clum, A., Copeland, A., Huddleston, J., Eichler, E. E., Turner, S. W., and Korlach, J. 2013. Nonhybrid, finished microbial genome assemblies from long-read SMRT sequencing data. Nat. Methods 10:563-569.

Dutta, B., Gitaitis, R., Agarwal, G., Coutinho, T., and Langston, D. 2018. Pseudomonas coronafaciens sp. nov., a new phytobacterial species diverse from Pseudomonas syringae. PLoS One 13:e0208271.
Elliot, C. 1920. Halo-blight of oats. J. Agric. Res. 19:139-172.

Griffiths, D. J., and Peregrine, W. T. H. 1956. Halo blight of oats. Plant Pathol. 5: 95-97.

Hunt, M., Silva, N. D., Otto, T. D., Parkhill, J., Keane, J. A., and Harris, S. R. 2015. Circlator: Automated circularization of genome assemblies using long sequencing reads. Genome Biol. 16:294.

Hwang, M. S., Morgan, R. L., Sarkar, S. F., Wang, P. W., and Guttman, D. S. 2005. Phylogenetic characterization of virulence and resistance phenotypes of Pseudomonas syringae. Appl. Environ. Microbiol. 71: 5182-5191. 
Kim, Y. C. 2020. First report of oat halo blight caused by Pseudmonas coronafaciens in South Korea. Plant Dis. Published online. 10.1094/PDIS-07-19-1519PDN

Koren, S., Walenz, B. P., Berlin, K., Miller, J. R., Bergman, N. H., and Phillippy, A. M. 2017. Canu: Scalable and accurate long-read assembly via adaptive $k$-mer weighting and repeat separation. Genome Res. 27:722-736.

Seemann, T. 2014. Prokka: Rapid prokaryotic genome annotation. Bioinformatics 30 : 2068-2069.
Seppey, M., Manni, M., and Zdobnov, E. M. 2019. BUSCO: Assessing genome assembly and annotation completeness. In: Gene Prediction. Methods in Molecular Biology, Vol. 1962. M. Kollmar, ed. Humana, New York.

Walker, B. J., Abeel, T., Shea, T., Priest, M., Abouelliel, A., Sakthikumar, S., Cuomo, C. A., Zeng, Q., Wortman, J., Young, S. K., and Earl, A. M. 2014. Pilon: An integrated tool for comprehensive microbial variant detection and genome assembly improvement. PLoS One 9:e112963.

Wilkie, J. P. 1972. Halo blight of oats in New Zealand. N. Z. J. Agric. Res. 15:461-468. 